\title{
Still seeking the missing patients with familial hypercholesterolemia
}

\section{Ansell D* \\ Institute of Applied Health Research, Birmingham University, UK}

In 2013 the European Atherosclerosis panel [1] highlighted the under diagnosis and under treatment of patients with heterozygous familial hypercholesterolaemia (heFH) in most, if not all, European countries. In the UK, NICE recently updated its clinical guideline (CG71) to primary care doctors for screening patients and identifying patients with heFH [2]. Are we any closer to knowing the true prevalence of heFH?

Using the infamous expression by Donald Rumsfeld there are known knowns (those recorded as having heFH) known unknowns (those who have had their cholesterol measured, but not labelled as heFH) and the unknown unknowns (do we know the population prevalence of heFH). The estimated prevalence of heFH within the $\mathrm{EU}$ ranges from the lower end prediction of 1:500 [1] to an upper end rate of 1:137 in a white Danish population cohort from the Copenhagen General Population Study [3] using the Dutch Lipid clinic Network (DLCN) criteria as definite / probable

Using the prevalence criteria of 1: 500 the EU panel calculated that the Netherlands had diagnosed $71 \%$ of the heFH population [1], while the UK had diagnosed only $12 \%$ giving a diagnosed prevalence rate of 1:700 and 1:4,160 respectively. In a study from Medway clinical commission group (CCG) to identify undiagnosed patients with heFH, in the study population of 262,000 , the initial heFH prevalence rate was 1:750 which increased to 1:375 at the end of the study period [4].

Why is all this so important? Firstly, patients with heterozygous familial hypercholesterolaemia have an increased risk of premature coronary heart disease and for any given LDL- cholesterol level their risk differs from risk charts for patients without heFH, in that their risk is from a cumulative high cholesterol from birth. This is why it is so important to identify these individuals early (primary patients without cardiovascular disease) and reduce their risk through lowering their lipid levels. In one series of patients with heFH, the risk of a coronary event by age 40,50 , and 65 years was $24 \%, 51 \%$ and $85 \%$ respectively [5]. In one study of first and second-degree relatives of heFH patients, the risk of fatal and nonfatal CHD by age 60 was $52 \%$ for male and $32 \%$ for female relatives [6].

Secondly, we need to know if our strategies are identifying some or all of the heFH population. Thirdly the differing screening programme strategies required to identify the heFH primary population will have cost and resource implications. Is there another way to estimate the prevalence in the heFH population without existing $\mathrm{CV}$ disease and who therefore may not have had their lipids measured? In the UK the primary care Quality Outcomes Framework incentivises GPs to ensure that all patients with diabetes have their lipids checked. Using UK primary care data from The Health Improvement network (THIN), the author has calculated the heFH prevalence rate of 1:210 of patients with diabetes AND who do not have a current / previous diagnosis of cardiovascular disease AND who have an existing recorded diagnosis of heFH. A study in the Netherlands [7] of the prevalence of type 2 diabetes in heFH patients $\mathrm{v}$ unaffected relatives showed that the prevalence of heFH in patients with type 2 diabetes was lower at $0.6: 1$. If we apply this reduced rate of heFH in patients with type 2 diabetes to the UK rate of heFH in patients with diabetes we would estimate a prevalence rate of heFH of 1:125 for the UK general population. This estimate aligns very closely with the figure of 1:137 from the Copenhagen study [3].

While it is relatively easy in a primary care database to identify patients with heFH who have already had their lipids measured by utilising an algorithm (DLCN or Simon Broome), identifying the remaining group is more difficult and resource intensive

The NICE guidance [2] advised GPs to screen and counsel all firstdegree relatives of patients with premature heart disease and referring the person to an FH specialist service for DNA testing if they meet the Simon Broome criteria for possible or definite $\mathrm{FH}$, or they have a DLCN score greater than 5 . If any are positive then carry out cascade testing using DNA testing to identify affected first- and second- and, when possible, third-degree biological relatives of people with a genetic diagnosis of $\mathrm{FH}$. To find these patients, consent from the index case would also be required. NICE indicates that of those genetically screened at one centre, $31 \%$ have a confirmed diagnosis [8].

Undertaking such a potential large population screening exercise in any country will require the resource of specialist personnel to identify, track, counsel and test people, in addition to the costs of laboratory testing of lipids and of genetic testing. In the UK no additional funding has been allocated or ring fenced for this, which will seriously impact on its implementation. Other EU countries also do not appear to have implemented the EU panel guidelines and its implementation will require a regional support infrastructure, in addition to provision of local resources.

\section{References}

1. Nordestgaard BG, Chapman MJ, Humphries SE, Ginsberg HN, Masana L, et al. (2003) Familial hypercholesterolaemia is underdiagnosed and undertreated in the genera population: guidance for clinicians to prevent coronary heart disease: Consensus Statement of the European Atherosclerosis Society. Eur Heart J34: 3478-3490. [Crossref]

2. Familial hypercholesterolaemia: identification and management. Available at: https:// www.nice.org.uk/guidance $/ \operatorname{cg} 71$

${ }^{*}$ Correspondence to: Ansell D, Academic Clinical Lecturer, Institute of Applied Health Research, Birmingham University, UK, E-mail: D.Ansell@bham.ac.uk

Received: September 04, 2018; Accepted: September 19, 2018; Published: September 24, 2018 
3. Benn M, Watts GF, Tybjaerg-Hansen A, Nordestgaard BG (2012) Familial hypercholesterolemiain the Danish general population: prevalence, coronary artery disease, and cholesterol-lowering medication. J Clin Endocrinol Metab 97: 3956-3964. [Crossref]

4. Green P, Neely D, Humphries SE3; Medway FH Audit Steering Committee (2016) Improving detection of familial hypercholesterolaemia in primary care using electronic audit and nurse-led clinics. J Eval Clin Pract 22: 341-348. [Crossref]

5. Slack J (1969) Risks of ischaemic heart-disease in familial hyperlipoproteinemic states. Lancet 2: 1380-1382. [Crossref]
6. Stone NJ, Levy RI, Fredrickson DS, Verter J (1974) Coronary artery disease in 116 kindred with familial type II hyperlipoproteinemia. Circulation 49: 476-488. [Crossref]

7. Besseling J, Kastelein JJ, Defesche JC, Hutten BA, Hovingh GK (2015) Association between familial hypercholesterolemia and prevalence of type 2 diabetes mellitus. JAMA 313: 1029-1036. [Crossref]

8. https://www.nice.org.uk/sharedlearning/familial-hypercholesterolaemia-geneticscreening-clinics

Copyright: $\odot 2018$ Ansell D. This is an open-access article distributed under the terms of the Creative Commons Attribution License, which permits unrestricted use, distribution, and reproduction in any medium, provided the original author and source are credited. 